\title{
Implications of Enuresis in Children and Their Families
}

\author{
Sefa Bulut, Thseen Nazir \\ Department of Counseling Psychology, Ibn Haldun University, Istanbul, Turkey \\ Email: sefabulut22@gmail.com, thseen.nazir@ihu.edu.tr
}

How to cite this paper: Bulut, S., \& Nazir, T. (2020). Implications of Enuresis in Children and Their Families. Open Journal of Depression, 9, 31-42.

https://doi.org/10.4236/ojd.2020.93004

Received: December 11, 2019

Accepted: July 26, 2020

Published: July 29, 2020

Copyright $\odot 2020$ by author(s) and Scientific Research Publishing Inc. This work is licensed under the Creative Commons Attribution International License (CC BY 4.0).

http://creativecommons.org/licenses/by/4.0/

\section{(c) (i) Open Access}

\begin{abstract}
Nocturnal enuresis (bedwetting during the night at sleep) is one of the most disturbing and frustrating childhood problems for both parents as well as children (Bulut, 2019). As many as 5 million American children and families are suffering from it (Warzak, 1993). Nocturnal enuresis is one of the most common disorders of childhood (Schulpen, 1997). If it is not treated, it has a significant effect on children's social and emotional health. Even when they reach adulthood, enuresis can still have a serious effect on their careers, social life and personal relationships (Graff, 1992). Furthermore, enuresis causes a financial drain in both the families and the society as a whole. Continuously having to wash, dry, and buy washing soaps and diapers can cause a significant financial burden on families. Additionally, enuresis can limit the family's mobility and vacation planning. Therefore, it seems that it has multiple consequences to be considered.
\end{abstract}

\section{Keywords}

Nocturnal, Enuresis, Childhood, Disorder, Burden of Enuresis, Family Implication of Enuresis

\section{Introduction}

"Enuresis" is not a new problem among children but is as old as humans are in this world and it is not specific to one race, region or community but prevails all across globe (Bulut, 2019). There are articles which go back as early as 1940's even. Later in 1950's enuresis was studied in depth and many researches had been done about nocturnal enuresis and etiologic aspects of the problem (Hallgren, 1958). Since then, there have been numerous publications about this topic and treatment solutions. Now, the scientific literature has accumulated huge amount of information, which provide very rich insights about the prob- 
lem at hand. Nocturnal enuresis (NE), commonly known as bed-wetting, is one of the most frequent pediatric disorders. Nocturnal enuresis is an involuntary urination that happens at night while sleeping, even after an age when a person should be able to control his or her bladder. Nocturnal enuresis can be defined as nighttime bedwetting in children five years of age or older (Nevéus et al., 2006). More specifically, primary nocturnal enuresis (PNE) is defined as the involuntary discharge of urine at night in children aged 5 years or older, who do not have congenital or acquired defects of the central nervous system or urinary tract and have not experienced a dry period of more than 6 months (Culbert \& Banez, 2008). It is not a serious health problem, and children usually outgrow it. Still, bedwetting can be upsetting for children and parents. Enuresis is a cause of social, psychological, and emotional distress and carries a significant clinical burden (Caldwell et al., 2013). It may or may not be associated with other lower urinary tract symptoms (LUTSs) and thus can be classified as Non-Monosymptomatic Nocturnal Enuresis (NMNE) or Monosymptomatic Nocturnal Enuresis (MNE), respectively. This leads to nights of disturbed sleep affecting a person's quality of life (QoL), thus causing mood disturbances, daytime sleepiness, fatigue, and reduced work productivity (Coyne et al., 2003). When MNE is diagnosed in children who have never achieved nocturnal urinary continence and have never been dry at night, it is defined as primary MNE (PMNE). On the other hand, secondary MNE (SMNE) describes children who have experienced a previous dry period of at least 6 months and it is usually secondary to psychological stress or organic causes such as urinary tract infections, obstructive sleep hypoventilation, diabetes mellitus/insipidus, and neurogenic bladder.

A diagnosis of nocturnal enuresis can be socially disruptive and psychologically stressful for a child. Quality of life indicators has been explored in the past to determine the impact of Nocturnal enuresis on the life of children. However, not a causal factor, poor school performance has been reported to be higher in children with Nocturnal enuresis (Sarici et al., 2016). A child's interpretation of the impact of Nocturnal enuresis on their own life also appears to vary from the parental proxy perception of quality of life (Bower et al., 2006). Parents have reported more often $(71 \%)$ that Nocturnal enuresis had little or no impact on their child's overall quality of life, as they believed it was a developmental condition (Sarici et al., 2016). This is supported by the fact that only half of the amount of parents consult a medical practitioner for this condition (Schlomer et al., 2013), which suggests that the prevalence could be higher in the community than indicated by current documented figures (Thurber, 2017).

The purpose of this study was to evaluate the implications of Enuresis in Children and Their Families as this condition can be stressful for the entire family.

\section{Family Implications of Enuresis}

Since psychology was seen as a professional job, enuresis was also seen as a psychological problem. Historically, enuresis was considered a severe personality 
problem. In early studies, some of the neurotic symptoms such as, somnambulism, nightmares and nail biting were considered as part of the enuretic spectrum. In psychiatric practice, these individuals are seen as immature, insecure and emotionally disturbed. Therefore, it was understood that psychotherapy was the only possible treatment (Levine, 1943). However, over the years this notion was discarded for a more medically oriented treatment option. This was considered maturational delay (Cederbald \& Rahim, 1986). Nowadays it is more often viewed as a maturational or a hereditary problem (Schulpen, 1997) or otherwise, a bio behavioral problem (Warzak, 1993).

Epidemiological studies from European, Asian and Middle East countries detailed almost similar reports with some minor differences, which might be attributed to the age of the research population and instruments used (Bulut, 2019). For example, In Turkey, Oge, Koçak and Gemalmaz (2001) reported 11.6\%, and Ozden et al. (2007) found 17.5\%. In another study in Turkey, Carman, Ceran, Kaya, Nuhoğlu and Karaman (2008) compared well-developed, affluent and other less affluent neighborhoods in the metropolitan areas of Istanbul and found $16 \%$ in wealthy parts and $25 \%$ in other parts of the city. Saudi Arabia, Kalo and Bella (1996) found the overall rate of $15 \%$. Similarly, in Taiwan, Cher, Lin and Hsu (2002) reported 5.5\%, Kanaheswari (2003) found 8\% among urban Malaysian elementary school children and claimed that their prevalence rates are lower than that of the developed countries. To sum it up, enuresis is a common chronic health problem, affecting about $10 \%$ of 7 -year-old children in developed countries (Pugner \& Holmes, 1997). Studying family risk factors in different cultures, might give us some culture specific hints for possible intervention programs (Cher et al., 2002). Child rearing and discipline practices in different societies might differ from each other, which might also affect the treatment of this problem. That also has to be taken into account when people from different cultural backgrounds are considered.

Morgan and Young (1975) reported that mothers from lower social classes and immigrants tend to be more intolerant; these mothers were also inpatient during the treatment process and were more likely to withdraw their children early on from treatment. Enuresis has been under reported and the parents of children with enuresis do not consider it as a serious problem (Readett, Bamigbade, \& Serjeant, 1991).

Most of the parents use different methods to ease the condition on their own; such as waking the child up from sleep to go to the toilet, fluid restriction after dinner and just talking about the problems. About 20\% - 36\% of parents used punishment for the bedwetting. This could develop severe tension between the parents and children; moreover, in severe cases children may be harassed or abused because of their bedwetting (Shelov et al., 1981). Similarly, in Turkey, Oge et al. (2001) reported that a great number of Turkish families do not consider enuresis a serious problem. The researchers observed that both parents and the physicians were quite ignorant about enuresis. This is due to the fact that in most of these cases, the parents themselves were ashamed of reporting their 
children's condition. Such as, in Malaysia, Kanaheswari (2003) found that $74 \%$ of parents felt very embarrassed and therefore, did not seek any medical help.

Kalo and Bella (1996)'s study in Saudi Arabia, reported the highest prevalence rates at the age of 7 around $24.5 \%$ and 11 years with $14.9 \%$ respectively. There are other studies reporting about $10 \%$ at the age of seven (Pugner \& Holmes, 1997). Many studies reported higher rates for boys than girls. It seems that the ranges gradually decrease, as the child gets older. However, there are studies reporting about $2 \%$ or $3 \%$ of enuresis at the age above 20 . Schulpen (1997) believed that about $1 \%$ to $3 \%$ of people above the age of 20 are still bedwetting. There are even studies that were conducted in military units reporting some enuresis cases in military service. However, Kalo and Bella (1996)'s study in Saudi Arabia, did not find enuresis cases at the age of 14 or 15. This could be because the parents were ashamed of reporting their children as an enuretic. This is an important point that needs to be taken into serious consideration. Obviously, it is a major health problem and it has social and economic implications for both the family and the community. Therefore, the purpose of this paper is aimed at dealing with the affect enuresis has on families.

Enuretic children think that they are the only ones with this problem and make a significant effort to hide the condition from their friends (Schulpen, 1997). The majority of them have never discussed their conditions outside of any family members (Friman \& Warzak, 1990). Enuretic children feel different and afraid of being detected by their friends. They often think that they are the only ones who have the problem (Schulpen, 1997). Because of their condition, they restrain themselves from overnight stays with their friends or going on school trips. In a British study by Butler, Redfern \& Forsythe (1990) children were mostly concerned about the social implications of bedwetting. They were bothered by the fact that they were not able to participate in social activities and overnight stays with their friends or go on school trips. They were anxious to change their beds and take a shower. Some number of children perceived family intolerance and sibling teasing.

Half of the enuretic children experience family intolerance and sibling teasing. According to Warzak (1993), enuretic children are more at risk for emotional and physical abuse from family members and they are afraid of being detected by their peers. Thus, their self-esteem is significantly affected by their condition. Studies show that enuretic children have lower self-esteem than healthy children. Even among chronic illnesses, enuresis has more negative effect on children's mental and social health (Hinde et al., 1995). Consequently, enuretic children may develop some psychosocial problems as a reaction to enuresis (Schulpen, 1997). This can lead to and create a vicious cycle through which children may suffer emotional and behavioral problems in many ways and forms.

They also face family intolerance and possible sibling harassment. This consequently affects their self-esteem and self-concept. The results of these studies show that, families are more tolerant of the younger children, as the child gets older parents tend to see it more as a personality trait (Butler, Brwein, \& For- 
sythe, 1986), in fact, it is not. Once the child gets older, the parents start to seek help. Mostly when the child gets around the age of 9, the family starts to seek serious medical treatment (Shelov et al., 1981).

Enuresis may cause significant social and economic burden on the family (Schulpen, 1997) and consequently causes an economic damage on the society. This leads to economic burden not only in the family, but also in the state health services and the state health insurances, which are also negatively affected (Morgan \& Young, 1975). There are many studies conducted with parents of enuretic children. In contrast to enuretic children reporting their conditions or experiences, there are more studies conducted with their parents. Mothers are especially chosen, their feelings and coping mechanisms with regards to the problem is then investigated (Schulpen, 1997).

Most of the parents think the bedwetting is an uncontrollable condition caused by lack of maturation, emotional problems and deep sleeping. This leads to the parents taking on a helpless or hopeless attitude towards the problem. They feel that the problem is untreatable and eventually develop a tolerant attitude towards their children's enuresis condition. Moreover, as the child gets older and the problem continues, mothers tend to become more intolerant and start to associate the condition with the child's personality (Butler, Brwein, \& Forsythe, 1986).

Norgaard and Anderson (1994) speculated that the real cost of enuresis for a family budget is underestimated and the financial difficulties constitute a significant burden on the family budget. Parental concerns included personal problems faced, a need for extra washing, expenditure and the smell (Butler, Brwein, \& Forsythe, 1986).

The financial difficulties and the added laundry chores restrict the social mobility of the family. Enuretic families may be unable to choose their holiday destinations freely and because of these problems, some families would prefer not to go on holidays to distant places (Jonge, 1969).

Although enuresis causes a significant increase in stress levels and poses a burden onto the child and the family, only 1 in 3 of them seek appropriate professional help in the USA (Sherman, He, Kalaydjian, Brothers, \& Merikangas, 2009). Surprisingly, similar attitudes were observed in other parts of the world. In Pakistan, only $36 \%$ of parents of enuretic children have consulted doctors, instead, they prefer other homeopathic treatments or anticipate the problem to disappear on its own (Mithani \& Zaidi, 2005). In India, similar patterns were also observed as De Sousa, Kapoor, Jagtap and Sen (2007) reported that the majority of parents with school going children were not concerned about their children's enuresis condition and only $26 \%$ of them sought help from medical doctors. In a Turkish study, Özden et al. (2007) found that only about $17 \%$ of children with enuresis were treated by physicians. The most common way of treatment was pharmacological ones. In another study conducted in urban Malaysia, Kanaheswari (2003) found that $87 \%$ of parents with enuretic children have never sought medical treatment. In this study, the significant predictors for 
enuresis in younger children were age, the male gender and having an Indian ethnic background. On the other hand, breastfeeding, being first-born, family integrity and stability were found to be protective factors, while home disruption, separation from family members and stressful life events before the age of 6 were found to be negative factors affecting the children. Furthermore, urinary tract infections, deep sleep, constipation and family problems seemed to negatively affect the children. Children who had experienced family separation and loss of loved ones, more likely the fathers, before the age of 6, were two times more likely to be enuretic than the children who belonged to an intact family constellation. The child is 4 times more likely to be enuretic if a history of family disruption and other psychosocial problems are present (Kalo \& Bella, 1996).

Morision (1998) postulated that enuresis might cause parents to experience anxiety and guilt, which in turn leads to incompetence in their parenting skills and abilities. This can result in problematic relationships with their children. Hence, it is imperative to provide educational and psychological support for parents and caregivers. This can alleviate the burden on their shoulders, and this way; they can fully concentrate on the treatment.

Most enuretic children are not aware that bedwetting is a common problem among children (Butler, Brwein, \& Forsythe, 1986). Besides, children do not know and recognize that enuresis is a medical condition and is nothing to be ashamed of. It is still an unexplored disorder (Karnicnic, Koren, Kos, \& Varda, 2012). This is the reason why children and their families do not apply for treatment. The same can be said for parents; they have very limited knowledge about the issue and spend their time waiting for the problem to be resolved naturally. To handle this inconvenience effectively, parental education and awareness is very important. Parents are the first agency between children and health care professionals; therefore, it is very essential for them to know the reasons, possible treatments and educational resources for the young members of their family. Most of the time, parents do not report any problems or seek professional help since they do not want to cause any embarrassment or they fear that their children would be labelled. They believe that as the child grows, the problems would disappear (De Sousa et al., 2007). Even in urban areas, in Malaysia, only 13\% would consult physicians about this problem (Kanaheswari, 2003). Similar figures were reported in Turkey as such that only $17 \%$ of enuresis children were treated by physicians (Özden et al., 2007). The authors concluded that somehow parents in Turkey do not pay attention to or understand the problem of enuresis; therefore, the majority of children do not receive appropriate or suitable treatment, even though these are available in medical institutions.

Literacy, education and awareness about enuresis are fundamental to overcoming the disorder. Research shows that children with enuresis belong mostly to the low socio-economic strata. Akis, Irgil and Aytekin (2002) conducted a study on Turkish children, the results of which revealed that the condition manifested more in children, whose mothers had less education or both parents were jobholders. In this study, enuresis was found to be more common in the 
first-born child.

The characteristics of enuretic parents were, large families, home disruptions, poverty, neurotic parents and siblings and lack of security in childhood (Levine, 1943). De Sousa et al. (2007) listed poor arousal, asphyxia, cesarean birth, low birth-weight, lack of breastfeeding, parents with health problems and living with stepparents as the main risk factors for nocturnal enuresis. In another study, in Taiwan, Cher et al. (2002) reported being of a younger age, male, a big family size, parenting style and parent's education level as possible family risk factors. Their results were comparable to Western societies. They also pointed out some cultural influences on the etiology and intervention programs. Kanaheswari (2003) also suggested studying multicultural communities in order to detect the effect of genetic ties or cultural upbringing on the development of enuresis.

\section{Family Cancellation of Enuresis Children}

There are also other studies conducted in different parts of the world concerning the problem of enuresis. Studies have reported that enuretic children come from a low socioeconomic level (De Sousa et al., 2007) and crowded families (Abul-Rahman, Hussain, \& Abdul-Rahman, 2009). Akis et al. (2002) observed that children with enuresis are usually the first-born ones. This may be because parents are not experienced enough to cater for the needs of their first child. On the contrary, Kalo \& Bella, 1996's study in Saudi Arabia found the reverse which they did not find common among the firstborns. Parents from low social classes are more likely to punish their children for bedwetting and blame them, which creates a vicious cycle, by aggravating the situation and cause tension between parents and children. Parents with limited education and cultural capacity are not able to understand the overall dynamics of the enuresis and tend to regard their children as having psychological problems. Enuresis is a treatable medical problem. Therefore, educating parents is necessary for timely diagnosis and treatment (Mithani \& Zaidi, 2005).

\section{Family Education}

Parents' attitude about treatment is crucial (Shelov et al., 1981). They may agree with the physician to continue the treatment or prematurely withdraw from a successful treatment plan (Schulpen, 1997). However, with appropriate techniques and treatments, both children and their families may learn to live with this condition and enjoy their life. Thus, physicians should realize that treatment of enuresis is important not only for the child but also for the whole family. After the successful treatment, the whole family can be relieved psychologically, have a better social life and have some financial improvements.

\section{Community Level Interventions}

It is safe to say that it is quite imperative to provide screening services in schools, kindergartens and community health centers. Teachers, school counselors and physicians as well as nurses are at an important position to take initiative regarding the solution of the problem and to provide education services for parents and children.

Developing Awareness and Assurances for Children and Parents 
Children should be assured that this is not their fault; on the contrary, it is a biological condition, which will disappear soon. Karnicnic et al. (2012) stated that many children do not know about their parents' and grandparents' enuresis history. Knowing that this condition was caused by genetic and biological factors could have provided them with an enormous sense of relief. This will help children feel better and relax. They will start feeling good about themselves and repair their self-esteem and self-image. This should be considered an educational and public health concern that all responsible parties should take part in, to contain the issue in a safe and healthy way.

Nowadays, most commonly pharmacological and behavioral interventions are suggested for the treatment of this problem (Warzak, 1993). In some cases, the combination of both is prescribed for patients in order to reach quick and effective treatment. Drug treatments involve giving daily desmopressin acetate (which is quite similar to antidiuretic hormone) (Nunes, O’Flynn, Evans, \& Sawyer, 2010). However, because of some side effects, such as, dry mouth, sleep disturbances and mood fluctuations some of the parents may oppose for that (Morris, 1993). This treatment can cost to family approximately 100 dollars for monthly. Thus, this can create a burden and this may not be covered by insurance companies (Ward-Smith \& Barry, 2006).

There are also other behavioral techniques used to control nighttime wedding. Parents can organize the children eating and fluid consumption in such a way that, children's fluid consumption can be stopped or reduced at an early time in the evening before bedtime. Controlling children drinking habits can be difficult and competitive. Along with these techniques, parents can wake up their children in the late midnights, one or two times. This can be also a burden on the parents since they themselves have to get up, wake up the child, and carry them to the bedrooms.

Behavioral modifications techniques involve using rewards for children and withholding rewards from children. Children can be given a chart of whole month, if the child wake up dry then he or she can draw a smiling sun shine for that day, and receive a reward for that day, or the children are asked to reach a dry period for a week then can get the rewards. The reverse is using the punishment, which is not effective and humanly methods that can cause counterproductive results.

An alarm system can be also used for prevention of urination. An small alarm apparat can be attach to children sheets or underwear when they wet the alarm start to ring then the child should be able to wake up and go to restroom. Parents and children needs to be taught how to use the alarm systems and use it appropriately. If the alarm systems do not work for the child then the medication can be considered as a last resort.

In recent years, electronic bladder diaries have started to become more efficient for the diagnosis and treatment of this condition. These diaries are child-centered and easy-to-keep. The traditional paper and pencil bladder diaries have poor completion rates. Thus, electronic bladder diaries have been 
proposed to overcome these difficulties (Myint, Adam, \& Herath, 2016).

\section{Conclusion}

To sum it up, enuresis has been established as a medical condition that is quite common and can be considered a universal phenomenon. The significance of this paper is to understand enuresis and its implications for child as well as for the family. The awareness and knowledge about enuresis is still limited among parents due to which children as well as parents suffer both. It not only affects the disease-ridden children but also the parents and the entire family, who end up living drastically. Despite the fact that the family tends to be secretive about the condition, they end up taking a huge toll financially, physically as well as mentally. It must be noted that the rates of enuresis, its causes and familial risks are quite prevalent in many countries. Therefore, some of these countries have taken it upon themselves to develop proper medical guidelines and treatment protocols for the treatment of enuresis. In addition, while the medical community has been working on this issue, the public should be aware of the implications of this condition on individuals and families as a whole. The public requires psycho-education on enuresis, its causes and treatment options, so that the stigma related to this condition will be eradicated. It appears that this topic needs to be opened up in public and discussed widely in media in order to draw public attention to develop awareness about the enuretic children and their families as well. Psycho-education must also be given to the professionals working in close proximity and who are in regular contact with the children. This could include school staffs, counselors and others. They need to know the reasons, risk factors, epidemiology, prevalence, and the available treatment models along with a concrete idea of where they can seek help from or if the case is beyond their capabilities, then where to refer these children. These individuals should be aware of the community resources, utilize them effectively so that the child can lead a peaceful, and condition free life. While considering the possible negative effects of enuresis on children, one must also consider the difficulties families go through as well. The families with an enuretic child end up becoming weak financially and in addition to this increase interpersonal tension among the family members. While psycho-educating families, focus should also be placed on the mental health of the family members who must have spent an exhaustive amount of time and energy on trying to cure or correct their child. Parent or family members need to be equipped with the required skills and coping mechanisms when dealing with an enuretic child. This would further ensure a smooth treatment process wherein both the enuretic child and the family are able to go on with their lives in a functionally sound manner. This paper also tries to understand that the enuresis phenomenon is common in childhood but researches had proved that in most of the cases, if followed with proper treatment, it can be overcome. However, the fact is that before overcoming this, many societies choose to hide the issue because of social or cultural barriers and 
not treating it the way it must be and let child and others suffer silently. As we had seen above that how big toil it takes so, the need of the time is to make people more aware about the issue and enlighten the concerned parties about the issue. In addition, the most important aim was to make people aware that such issue needs to be handled at proper time with proper resources instead of suffering silently or using age-old remedies.

\section{Conflicts of Interest}

The authors declare no conflicts of interest regarding the publication of this paper.

\section{References}

Abul-Rahman, S., Hussain, R., \& Abdul-Rahman, S. (2009). Prevalence of Bedwetting for Children in Mosul City. Jordanian Medical Journal, 43, 44-50.

Akis, N., Irgil, E., \& Aytekin, N. (2002). Enuresis and the Effective Factors. A Case-Control Study. Scandinavian Journal of Urology and Nephrology, 36, 199-203. https://doi.org/10.1080/003655902320131875

Bower, W. F., Sit, F. K., Bluyssen, N., Wong, E. M., \& Yeung, C. K. (2006). PinQ: A Valid, Reliable and Reproducible Quality-of-Life Measure in Children with Bladder Dysfunction. Journal of Pediatric Urology, 2, 185-189.

https://doi.org/10.1016/j.jpurol.2005.07.004

Bulut, S. (2019). Enuresis: The Hidden Problem. Psychology and Psychotherapy Research Studies, 2, 1-3. https://doi.org/10.31031/PPRS.2019.02.000540

Butler, R. J., Brwein, C. R., \& Forsythe, W. I. (1986). Maternal Attributions and Tolerance for Nocturnal Enuresis. Behavioral Research and Therapy, 24, 307-312. https://doi.org/10.1016/0005-7967(86)90190-7

Butler, R. J., Redfern, E. J., \& Forsythe, W. (1990). The Child's Constructing of Nocturnal Enuresis and the Prediction of Effectiveness Using Pretreatment Variables. Journal of Child Psychology and Psychiatry, 31, 447-454. https://doi.org/10.1111/j.1469-7610.1990.tb01581.x

Caldwell, P. H., Deshpande, A. V., \& Von Gontard, A. (2013). Management of Nocturnal Enuresis. British Medical Journal, 347, f6259. https://doi.org/10.1136/bmj.f6259

Cederbald, M., \& Rahim, S. I. A. (1986). Epidemiology of Nocturnal Enuresis in a Part of Khartoum, Sudan. II. The Intensive Study. Acta Paediatrica, 75, 1021-1027. https://doi.org/10.1111/j.1651-2227.1986.tb10334.x

Cher, T. W., Lin, G. J., \& Hsu, K. H. (2002). Prevalence of Nocturnal Enuresis and Associated Familial Factors in Primary School Children in Taiwan. The Journal of Urology, 168, 1142-1146. https://doi.org/10.1097/01.ju.0000025872.38494.43

Coyne, K. et al. (2003). The Prevalence of Nocturia and Its Effect on Health-Related Quality of Life and Sleep in a Community Sample in the USA. BJU International, 92, 948-954. https://doi.org/10.1111/j.1464-410X.2003.04527.x

Culbert, T. P., \& Banez, G. A. (2008). Wetting the Bed: Integrative Approaches to Nocturnal Enuresis. EXPLORE, 4, 215-220. https://doi.org/10.1016/j.explore.2008.02.014

De Sousa, A., Kapoor, H., Jagtap, J., \& Sen, M. (2007). Prevalence and Factors Affecting Enuresis amongst Primary School Children. Indian Journal of Urology, 23, 354-357. https://doi.org/10.4103/0970-1591.36703 
Friman, P. C., \& Warzak, W. J. (1990). Nocturnal Enuresis: A Prevalent, Persistent yet Curable Parasomnia. Pediatrician, 17, 38-45.

Graff, M. J. M. (1992). 40 Years of Being Treated for Nocturnal Enuresis. The Lancet, 340, 957-958. https://doi.org/10.1016/0140-6736(92)92828-4

Hallgren, B. (1958). Nocturnal Enuresis; Aetiologic Aspects. Acta Paediatrica, 48, 66-74. https://doi.org/10.1111/j.1651-2227.1959.tb17503.x

Hinde, M. et al. (1995). Poor Self-Esteem in Children with Enuresis. Läkartidningen, 92, 3225-3229.

Jonge, A. (1969). Children with Enuresis, an Epidemiological and Clinical Study. Assen.

Kalo, B. B., \& Bella, H. (1996). Enuresis: Prevalence and Associated Factors among Primary School Children in Saudi Arabia. Acta Paediatrica, 85, 1217-1222. https://doi.org/10.1111/j.1651-2227.1996.tb18232.x

Kanaheswari, Y. (2003). Epidemiology of Childhood Nocturnal Enuresis in Malaysia. Journal of Pediatrics and Child Health, 39, 118-123. https://doi.org/10.1046/j.1440-1754.2003.00105.x

Karnicnic, K., Koren, A., Kos, N., \& Varda, N. M. (2012). Prevalence and Quality of Life of Slovenian Children with Primary Nocturnal Enuresis. International Journal of Nephrology, 2012, Article ID: 509012. https://doi.org/10.1155/2012/509012

Levine, A. (1943). Enuresis in the Navy. The American Journal of Psychiatry, 100, 320-325.

Mithani, S., \& Zaidi, Z. (2005). Bed-Wetting in School Children of Karachi. Journal of Pakistan Medical Association, 55, 2-5.

Morgan, R. T. T., \& Young, G. C. (1975). Parental Attitudes and the Conditioning Treatment of Childhood Enuresis. Behavioral Research Therapy, 13, 197-199. https://doi.org/10.1016/0005-7967(75)90018-2

Morision, J. M. (1998). Parents' and Young People's Attitude towards Bedwetting and Their Influence on Behavior, Including Readiness to Engage in and Persist with Treatment. British Journal of Urology, 81, 56-68. https://doi.org/10.1046/j.1464-410x.1998.00010.x

Morris, N. (1993). Agency for Health Care Policy and Research. AHCPR Guideline: Urinary Incontinence. Ohio Nurses Review, 68, 10.

Myint, M., Adam, A., \& Herath, S. (2016). Mobile Phone Applications in Management of Enuresis: The Good, the Bad, and the Unreliable! Journal of Pediatric Urology, 12, 112-116. https://doi.org/10.1016/j.jpurol.2015.09.011

Nevéus, T., von Gontard, A., Hoebeke, P., Hjalmas, K., Bauer, W., Jorgensen, T. M. et al. (2006). The Standardization of Terminology of Lower Urinary Tract Function in Children and Adolescents: Report from the Standardization Committee of the International Children's Continence Society. Journal of Urology, 176, 314-324. https://doi.org/10.1016/S0022-5347(06)00305-3

Norgaard, J. P., \& Anderson, T. M. (1994). Nocturnal Enuresis: A Burden on Family Economy? Scandinavian Journal of Urology and Nephrology, Supplementum, 163, 49-54.

Nunes, V. D., O’Flynn, N., Evans, J., \& Sawyer, L. (2010). Management of Bedwetting in Children and Young People: Summary of NICE Guidance. British Medical Journal, 341, c5399. https://doi.org/10.1136/bmj.c5399

Oge, O., Koçak, I., \& Gemalmaz, H. (2001). Enuresis: Point Prevalence and Associated Factors among Turkish Children. The Turkish Journal of Pediatrics, 43, 38-43.

Özden, C., Ozdal, O. L., Altinova, S., Oguzulgen, I., Urgancioglu, G., \& Memis, A. (2007). Prevalence and Associated Factors of Enuresis in Turkish Children. International Bra- 
zilian Journal of Urology, 33, 216-222.

https://doi.org/10.1590/S1677-55382007000200013

Pugner, K., \& Holmes, J. (1997). Nocturnal Enuresis: Economic Impacts and Self-Esteem Preliminary Research Results. Scandinavian Journal of Urology and Nephrology, Supplementum, 183, 65-69.

Readett, D. R., Bamigbade, T., \& Serjeant, G. R. (1991). Nocturnal Enuresis in Jamaican Children-Implication for Therapy. West Indian Medical Journal, 40, 181-184.

Sarici, H. et al. (2016) Prevalence of Nocturnal Enuresis and Its Influence on Quality of Life in School-Aged Children. Journal of Pediatric Urology, 12, 159.e1-159.e6. https://doi.org/10.1016/j.jpurol.2015.11.011

Schlomer, B., Rodriguez, E., Weiss, D., \& Copp, H. (2013). Parental Beliefs about Nocturnal Enuresis Causes, Treatments, and the Need to Seek Professional Medical Care. Journal of Pediatric Urology, 9, 1043-1048. https://doi.org/10.1016/j.jpurol.2013.02.013

Schulpen, T. W. (1997). The Burden of Nocturnal Enuresis. Acta Paediatrica, 86, 981-984. https://doi.org/10.1111/j.1651-2227.1997.tb15183.x

Shelov, S. P., Gundy, J., Weiss, J. C., Steven, P., Mclntire, J. M. S., Olness, K. et al. (1981). Enuresis: A Contrast of Attitudes of Parents and Physicians. Pediatrics, 67, 707-710.

Sherman, S,. He, J., Kalaydjian, A., Brothers, S., \& Merikangas, K. R. (2009). Prevalence of Enuresis and Its Associations with Attention Deficit Hyperactivity Disorder among USA Children: Results from a Nationally Represented Sample. Journal of American Academy of Child and Adolescence Psychiatry, 48, 35-41. https://doi.org/10.1097/CHI.0b013e318190045c

Thurber, S. (2017). Childhood Enuresis: Current Diagnostic Formulations, Salient Findings, and Effective Treatment Modalities. Archives of Psychiatric Nursing, 31, 319-323. https://doi.org/10.1016/j.apnu.2016.11.005

Ward-Smith, P., \& Barry, D. (2006). The Challenges of Treating Enuresis. Urologic Nursing, 26, 222-224.

Warzak, W. J. (1993). Psychological Implications of Nocturnal Enuresis. Clinical Pediatrics, 32, 38-40. https://doi.org/10.1177/0009922893032001S09 